\title{
Magnetic resonance imaging-guided laser interstitial thermal therapy as treatment for intractable insular epilepsy in children
}

\author{
M. Scott Perry, MD, ${ }^{1}$ David J. Donahue, MD, ${ }^{1}$ Saleem I. Malik, MD, ${ }^{1}$ Cynthia G. Keator, MD, ${ }^{1}$ \\ Angel Hernandez, MD, ${ }^{1}$ Rohit K. Reddy, MD, ${ }^{2}$ Freedom F. Perkins Jr., MD, ${ }^{2}$ Mark R. Lee, MD, PhD, ${ }^{2,4}$ \\ and Dave F. Clarke, MD2,3 \\ ${ }^{1}$ Comprehensive Epilepsy Program, Jane and John Justin Neuroscience Center, Cook Children's Medical Center, Fort \\ Worth; ${ }^{2}$ Comprehensive Epilepsy Program, Dell Children's Hospital, Austin; and Departments of ${ }^{3}$ Pediatrics and ${ }^{4}$ Surgery and \\ Perioperative Services, Dell Medical School, University of Texas, Austin, Texas
}

\begin{abstract}
OBJECTIVE Seizure onset within the insula is increasingly recognized as a cause of intractable epilepsy. Surgery within the insula is difficult, with considerable risks, given the rich vascular supply and location near critical cortex. MRIguided laser interstitial thermal therapy (LiTT) provides an attractive treatment option for insular epilepsy, allowing direct ablation of abnormal tissue while sparing nearby normal cortex. Herein, the authors describe their experience using this technique in a large cohort of children undergoing treatment of intractable localization-related epilepsy of insular onset.

METHODS The combined epilepsy surgery database of Cook Children's Medical Center and Dell Children's Hospital was queried for all cases of insular onset epilepsy treated with LiTT. Patients without at least 6 months of follow-up data and cases preoperatively designated as palliative were excluded. Patient demographics, presurgical evaluation, surgical plan, and outcome were collected from patient charts and described.

RESULTS Twenty patients (mean age 12.8 years, range 6.1-18.6 years) underwent a total of 24 LiTT procedures; $70 \%$ of these patients had normal findings on MRI. Patients underwent a mean follow-up of 20.4 months after their last surgery (range 7-39 months), with 10 (50\%) in Engel Class I, 1 (5\%) in Engel Class II, 5 (25\%) in Engel Class III, and 4 $(20 \%)$ in Engel Class IV at last follow-up. Patients were discharged within 24 hours of the procedure in $15(63 \%)$ cases, in 48 hours in $6(24 \%)$ cases, and in more than 48 hours in the remaining cases. Adverse functional effects were experienced following 7 (29\%) of the procedures: mild hemiparesis after 6 procedures (all patients experienced complete resolution or had minimal residual dysfunction by 6 months), and expressive language dysfunction after 1 procedure (resolved by 3 months).

CONCLUSIONS To their knowledge, the authors present the largest cohort of pediatric patients undergoing insular surgery for treatment of intractable epilepsy. The patient outcomes suggest that LiTT can successfully treat intractable seizures originating within the insula and offers an attractive alternative to open resection. This is the first description of LiTT applied to insular epilepsy and represents one of only a few series describing the use of LiTT in children. The results indicate that seizure reduction after LiTT compares favorably to that after conventional open surgical techniques. https://thejns.org/doi/abs/10.3171/2017.6.PEDS17158
\end{abstract}

KEY WORDS insular epilepsy; laser interstitial thermal therapy; epilepsy surgery

I NTRACTABLE epilepsy with seizures originating in the insula and nearby perisylvian cortex is increasingly recognized, due in part to improved recognition of insular seizure semiology. Isnard et al. described the semiology and electroencephalography (EEG) features in patients with insular seizures, a stereotyped sequence of ictal symptoms including laryngeal constriction, pares- thesia covering a large cutaneous territory, dysarthria, and focal motor convulsions. ${ }^{9}$ Data obtained from direct cortical stimulation of the insula supported the origin of these symptoms, and the authors concluded that the "sequence of ictal symptoms seemed reliable enough to characterize insular lobe epileptic seizures." However, stereo-electroencephalography (SEEG) exploration in patients with focal

ABBREVIATIONS AED = antiepileptic drug; EEG = electroencephalography; fMRI = functional MRI; LiTT = laser interstitial thermal therapy; MEG = magnetoencephalography; SEEG = stereo-EEG

SUBMITTED March 27, 2017. ACCEPTED June 28, 2017

INCLUDE WHEN CITING Published online October 13, 2017; DOI: 10.3171/2017.6.PEDS17158. 
insular involvement has often revealed wider involvement in the epileptogenic zone, including frontal and central regions. Seizure semiology in these cases varied greatly, with an evolution from subtle seizures, spasms, and myoclonus to asymmetrical tonic and hypermotor events. ${ }^{5}$

Access to the insula is difficult and fraught with potential for collateral injury due to its deep location and rich vascular supply. The insula is nestled within the sylvian fissure between the frontotemporal opercula and basal ganglia. Its surface is draped by the $\mathrm{M}_{2}$ segments of the middle cerebral artery, bearing long and short perforators, some which also perfuse the corona radiata..$^{15}$ The variety of proposed surgical approaches to invasive monitoring and resective epilepsy surgery within the insula attests to the trepidation (and dissatisfaction with available techniques) with which neurosurgeons approach the insula, especially in the dominant hemisphere. Its location and extensive connection with the rest of the brain often makes EEG evaluation challenging, with widespread or poorly localized onset using scalp EEG. In fact, insular onset has often been implicated as a reason behind failed epilepsy surgeries after frontal, temporal, or parietal lobe resection. ${ }^{13}$

Invasive monitoring techniques addressing the insula include freehand electrode placement, ${ }^{12}$ Bouthillier's "hybrid" opercular insular electrode, ${ }^{2}$ and oblique stereotactically directed insertion of depth electrodes. ${ }^{1}$ This last approach, supplemented by orthogonal leads to explore the midinsula, may provide the most detailed coverage. With respect to insular resective surgery, some surgeons employ a transopercular corridor to avoid vasospasm attributed to injury of conducting and perforating vessels associated with dissection in the sylvian fissure. ${ }^{8}$ Others split the sylvian fissure widely to allow either open freehand depth electrode placement or "pure" insular resection, apparently discounting the effects of dissection and retraction of opercular cortex and related blood vessels. In 1964, Silfvenius et al. reviewed the history of insular resection and noted the "considerably higher" rate of complications (20.6\% vs $2.8 \%$ ) when temporal lobectomy included insular resection. ${ }^{14}$ As a result, methods of surgical therapy for intractable insular epilepsy that avoid these potential dangers are worthwhile.

MRI-guided laser interstitial thermal therapy (LiTT) is a minimally invasive stereotactic surgical technique that can be employed to apply laser-induced heat to ablate epileptogenic foci. Application of LiTT for the surgical treatment of epilepsy has been largely restricted to lesional temporal lobe epilepsy, with limited data on morbidity and seizure outcome following extratemporal laser ablation. ${ }^{10,11,17}$ The minimally invasive nature of LITT, along with its ability to provide real-time ablation monitoring and avoid collateral damage, suggests that thermal ablation has the potential to improve the surgical management of intractable insular epilepsy in appropriately selected candidates. We have used LiTT for the treatment of intractable pediatric epilepsy since 2013 and herein describe our experience with patients with insular epilepsy to date.

\section{Methods}

We queried our comprehensive epilepsy surgery data- base for all cases in which LiTT was performed within the insula. We excluded patients with less than 6 months of follow-up, those for whom follow-up was not available, and patients undergoing preoperatively designated palliative procedures. Baseline patient characteristics were collected, along with results of presurgical EEG and imaging evaluations. The study protocol was approved by the institutional review boards of both the Cook Children's Medical Center and Dell Children's Hospital with waiver of consent granted. All patients underwent preoperative 3-T, T1-weighted thin-slice (1-mm) MRI before and after administration of contrast. Video EEG data were available for all patients. Additional functional imaging, including SPECT, PET, and magnetoencephalography (MEG), was performed at the discretion of the treating epileptologist. Hemispheric language dominance was determined using either functional MRI (fMRI) or MEG with receptive and expressive language paradigms. If results of one modality were inconclusive of language localization, the alternative modality was completed. In cases in which neither fMRI nor MEG convincingly localized language dominance, Wada or cortical mapping using subdural grids was performed. Patient cases were reviewed, and surgical plans were formulated during a multidisciplinary epilepsy surgery conference of assembled epileptologists, neurosurgeons, neuroradiologists, and neuropsychologists. For patients without convincing localization based on preoperative noninvasive evaluation, extraoperative EEG monitoring was completed, incorporating either stereotactically placed depth electrodes or a combination of subdural grids and depth electrodes to better characterize the epileptogenic zone. Operative reports, along with intra- and postoperative MR images, documented the extent of insular ablation for each patient and were categorized as primarily anterior, posterior, or both. Postoperative clinic visit notes were reviewed to record outcome, which was defined according to Engel criteria. ${ }^{6}$ In cases in which initial ablation failed and the patient returned for a second procedure, outcome for the initial surgery was recorded as Engel Class IV for all time periods after the second surgery. Outcome is presented both for individual surgeries and for individual patients. For patients in whom initial LiTT failed, MRI was repeated and coregistered to prior preoperative imaging to evaluate completeness of ablation within the targeted epileptogenic zone. After review, patients returned for repeat LiTT procedures targeting the remaining epileptogenic zone.

\section{Surgical Technique}

For patients requiring invasive monitoring with depth electrodes, placement was performed using one of 2 techniques. For low lateral or multiple trajectories we prefer a technique employing robot assistance (ROSA, Medtech) similar to that described by González-Martínez et al.7 Stereotactic planning for insular coverage usually involved 2 electrode trajectories. One of these trajectories started in the occipital region and was designed to cover the entire length of either the posterior or anterior long gyrus (Fig. 1D, G, and I). Thus, the electrode trajectory passed through the insular cortex from posterior to anterior, deep to the middle cerebral vessels, and superior to the exter- 


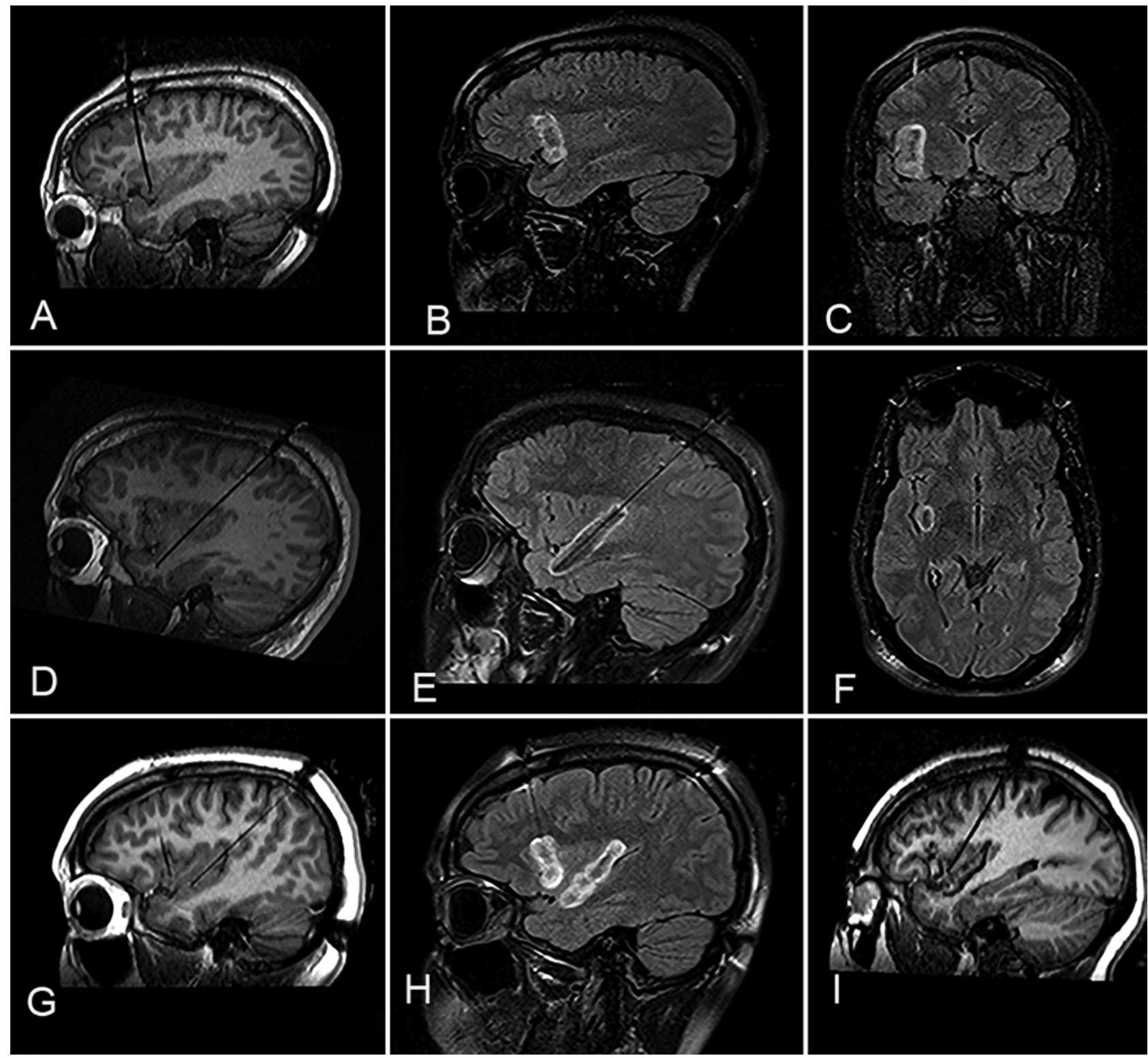

FIG. 1. Sagittal T1-weighted multiplanar reconstruction MR images obtained in 3 patients (1 patient per row) confirming laser fiber placement for the superior approach to the anterior insula (A), occipital approach to the posterior insula (D and I), and both approaches together (G). Postablation sagittal $(B, E$, and $H)$, coronal $(C)$, and axial $(F)$ T2-weighted FLAIR images demonstrating region of thermal ablation in the anterior $(\mathrm{B}, \mathrm{C}$, and $\mathrm{H})$ and posterior $(\mathrm{E}, \mathrm{F}$, and $\mathrm{H})$ insulae.

nal capsule. For the other trajectory, used in some cases, a frontal approach to the anterior insula from superior was employed, with the occipital approach targeting the posterior insula (Fig. 1A).

In some cases, subdural grid electrodes were used in addition to depth electrodes for monitoring the perisylvian cortex. In the same operative session, after placement of the depth electrodes, the Leksell frame was removed, and a Mayfield head holder was applied. The patient was positioned supine with a large shoulder roll, and the Mayfield head holder was attached to the operating table, with the vertex of the head neutral and the nasion slightly up. Typically, a large frontotemporal incision was made with the anterior fascia of the temporalis muscle brought with the skin flap dissection to protect the frontalis branch of the facial nerve. The temporalis muscle was incised at the anterior and posterior aspects of the exposure, and an osteoplastic bone flap was fashioned. The bone flap was retracted inferiorly, and the dura was opened. Electrocorticography was performed, and placement of the subdural grid electrodes was determined. Following closure of the dura and replacement of the bone flap, the electrode tails were tunneled out of separate stab wounds, and a JP drain was left in the subgaleal space. After recovering from anesthesia, patients were brought directly to the monitoring unit, and monitoring was started immediately. EEG monitoring continued until acquisition of adequate data was complete, patient data were again reviewed in a multidisciplinary con- 
ference, and surgical plans were discussed. Patients treated at Dell Children's Comprehensive Epilepsy Program routinely immediately undergo LiTT following SEEG using the same trajectory of the depth electrodes that define the epileptogenic zone. Patients at Cook Children's Medical Center typically return 6-8 weeks after SEEG for LiTT using similar trajectories modified in select cases to allow for ablation of epileptogenic tissue characterized by multiple leads with a single laser fiber pass.

Surgical ablation of the insula was carried out using the Visualase system (Medtronic) according to previously described methods. ${ }^{4}$ An operative plan for the laser fiber trajectory was fashioned using the same sequence for electrode trajectory planning.

During the lesioning process, we employed the imaging parameters and application of laser application as discussed elsewhere. ${ }^{4,17}$ For insular ablation, we generally use the 3-mm diffuser tip catheter. Temperature safety limits are entered to protect structures medial to the insula. Effective doses (generally 6-9 W applied for 90-128 seconds) to produce target temperatures approaching $90^{\circ} \mathrm{C}$ were applied at 4- to 6-mm intervals to ensure adequate, controlled lesioning. Lesion extent was assessed during ablation by observing the graphic "estimation of thermal ablation" provided by the Visualase system and temperatures at the preselected monitoring locations, generally corresponding to adjacent eloquent areas at risk. After completion of the ablation, as suggested by these parameters, lesion configuration was further assessed using MRI including FLAIR (Fig. 1B, C, E, F, and H), diffusion-weighted imaging, and T1-weighted postcontrast sequences. Patients were hospitalized overnight and received perioperative doses of dexamethasone, which were tapered over 7-21 days.

\section{Results \\ Patient Characteristics}

Between January 2013 and September 2016, 26 patients with intractable localization-related epilepsy underwent 31 LiTT procedures in which the insula was the surgical target. Six patients (7 procedures) were excluded, 4 patients did not return for follow-up after surgery and lacked follow-up data, and 2 were receiving palliative treatment. The 20 remaining patients underwent 24 LiTT procedures; 4 were repeat ablations after a failed initial ablation procedure. The average age at surgery was 12.8 years (range 6.1-18 years) with epilepsy onset at a mean age of 5.8 years (range 2 weeks-14 years). Patients were receiving an average of 2.2 antiepileptic drugs (AEDs) (range 1-4) at surgery after an average of 3.8 AEDs (range 1-6) had failed. All patients had localization-related epilepsy with 1-3 semiologies reported at the time of evaluation. Seizure occurrence was daily in 14 patients (70\%), weekly in 3 patients (15\%), and monthly in 3 patients $(15 \%)$. Neurological examination findings were normal in 18 patients (90\%); the remaining 2 patients had mild hemiparesis at baseline. Sixteen patients $(80 \%)$ were left-hemisphere dominant, 1 was right-hemisphere dominant, and 3 had bilateral language representation. Language was localized using either MEG or fMRI in 9 patients (45\%) and both modalities in 8 patients (40\%); 2 (10\%) patients underwent
Wada testing in addition to fMRI or MEG, and 1 patient's language $(5 \%)$ was localized using cortical stimulation during invasive EEG monitoring. Ten patients $(50 \%)$ had previously undergone failed open resections: 6 failed frontal lobectomies/corticectomies ( 2 including portions of the anterior insula), 2 failed superior temporal gyral/insular resections, 1 failed temporal lobectomy, and 1 failed parietal corticectomy. For patients who had undergone prior open resection, the pathology was cortical dysplasia in 9 patients and nonspecific changes in 1 patient.

All patients underwent MRI, which revealed lesional epilepsy with suspected malformations of cortical development in $6(30 \%)$ patients. Seizures were captured using scalp video EEG in all but 2 patients (90\%), although subsequent extraoperative monitoring captured seizures in those without ictal scalp EEG data. In $12(60 \%)$ patients, scalp EEG localized hemispheric onset within the frontal or temporal region. In the remaining patients, scalp EEG was interpreted to show bilateral/generalized onset or unclear onset. Other workup varied, with $19(95 \%)$ patients undergoing MEG, 7 (35\%) undergoing PET, 13 (65\%) undergoing ictal/interictal SPECT with subtraction, 2 (10\%) Wada, and 14 (70\%) fMRI. Results of the presurgical evaluation are presented in Table 1. After presurgical evaluation, 14 (70\%) patients underwent further invasive EEG monitoring: $11(79 \%)$ with stereotactically placed depth electrodes and $3(21 \%)$ with a combination of subdural grids/strips and depth electrodes. Six patients did not require invasive EEG monitoring prior to LiTT, lesions were noted on MRI and at least 1 additional congruent functional imaging study in 6, and 3 had MEG with tightly clustered interictal dipoles congruent with semiology and EEG.

Of the 24 LiTT procedures, $11(46 \%)$ were performed on the left side. Nine (38\%) procedures were performed in the dominant hemisphere, all left. The LiTT procedure was restricted to the insula in $13(65 \%)$ patients and included additional ablation of the opercular surface in 7 $(35 \%)$. Ablations were restricted to the anterior insula in $11(55 \%)$ patients, posterior insula in $4(20 \%)$ patients, and both regions in 5 (25\%) patients. Seven (35\%) patients underwent LiTT immediately after SEEG using the same fiber trajectory. Ablations were carried out with 1 fiber pass in 16 procedures; all others required 2 . We did not collect samples for pathology in any case.

\section{Seizure Response}

The mean follow-up after the last surgery was 20.4 months (range 7-39 months). According to Engel classification at the last follow-up, $10(50 \%)$ patients were in Class I, $1(5 \%)$ was in Class II, 5 (25\%) were in Class III, and $4(20 \%)$ were in Class IV. For patients with at least 1 year of follow-up $(\mathrm{n}=18), 9(47 \%)$ were in Engel Class I, $3(16 \%)$ were in Class II, $3(16 \%)$ were in Class III, and 3 $(16 \%)$ were in Class IV. One patient has not yet returned for the 1-year follow-up. At 2 years $(n=8), 4(50 \%)$ were in Engel Class I, 1 (13\%) was in Class II, and 3 (38\%) were in Class III.

Outcome was also evaluated for individual procedures. At the 6-month follow-up $(\mathrm{n}=24)$, patients in 10 cases $(42 \%)$ were in Engel Class I, those in 5 cases $(21 \%)$ were in Class II (rare seizures), and those in 4 cases (17\%) were 


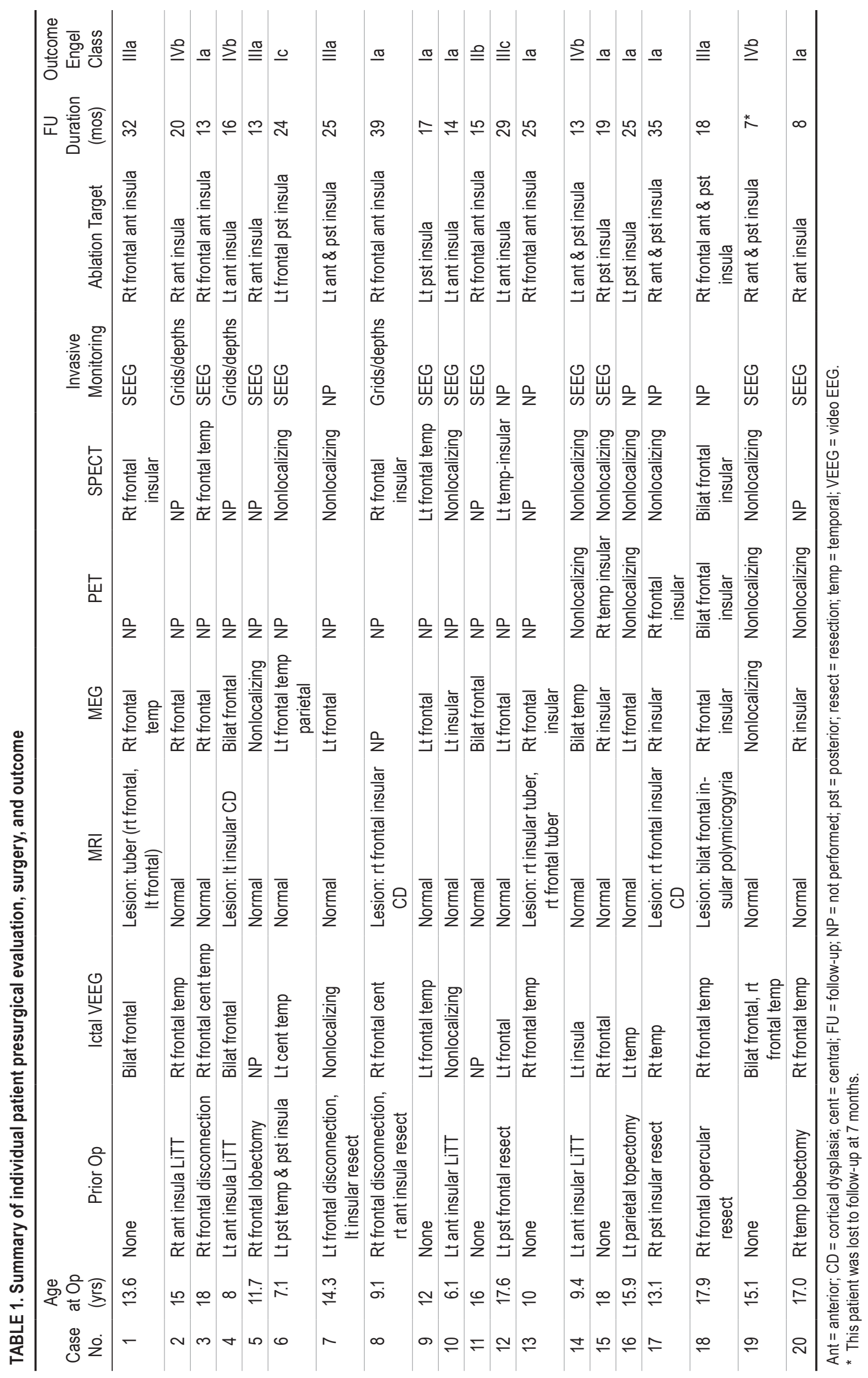


in Class III (worthwhile reduction). Patients in only 5 cases $(21 \%)$ reported no improvement in seizure frequency (Engel Class IV). One-year data were available for 22 procedures; the patient in 1 case had not yet reached the 1-year postoperative time point, and another patient had not returned for follow-up. At 1 year, patients in 9 cases $(41 \%)$ were seizure free, $1(5 \%)$ patient reported only rare seizures (Engel Class II), those in 5 cases (22\%) reported worthwhile reduction (Class III), and patients in 7 cases (32\%) reported no significant improvement. Patients who underwent 10 total procedures had more than 2 years of follow-up; those who underwent 4 procedures $(40 \%)$ remained seizure free, the patient in 1 case $(10 \%)$ had only rare seizures, patients in 3 cases $(30 \%)$ reported worthwhile improvement, and those in 2 cases $(20 \%)$ reported no improvement. Patients were receiving 27\% fewer AEDs following surgery and 4 had completely weaned off AEDs and remained seizure free.

\section{Hospitalization and Adverse Events}

Patients remained hospitalized an average of 1.8 days after surgery (range 1-10 days). Patients were discharged within 24 hours of the procedure in $15(63 \%)$ cases, in 48 hours in $6(24 \%)$ cases, and $>48$ hours in the remaining cases. One patient remained hospitalized 10 days after the procedure because rehabilitation was needed for postoperative weakness. Only 1 patient was readmitted within 30 days of surgery because of hypertension and desaturation in sleep unrelated to surgery, which resolved within days. Adverse functional effects were experienced following 7 (29\%) of the procedures. Of the 7 patients who underwent these procedures, 6 experienced mild hemiparesis ( 3 after anterior and 3 after posterior insular ablation, all completely resolved or were associated with minimal residual dysfunction by 6 months) and 1 experienced expressive language dysfunction (following anterior insular ablation, resolved by 3 months). Patients required nonnarcotic oral agents for pain control after $8(30 \%)$ procedures and brief narcotic pain control without narcotics at discharge in 14 (58\%) procedures. Only $3(13 \%)$ patients required narcotic pain control after discharge.

\section{Discussion}

In this series, to our knowledge we present the largest cohort of pediatric patients undergoing insular surgery for the treatment of intractable epilepsy to date. While the number of insular surgeries over the 3-year study period was significant, this was largely due to increased recognition of insular epilepsy and an increased willingness to monitor and perform surgery in the insula using SEEG and LiTT. Ten procedures were performed to reexamine previous surgeries that had failed, as insular onset is increasingly recognized as an etiology for failed temporal, frontal, or parietal resection. ${ }^{13}$ Our experience suggests that LiTT can be used to successfully treat intractable epilepsy originating within the insula and offers an attractive alternative to open resection. To our knowledge, this is the first description of LiTT applied to insular epilepsy and represents one of only a few series describing the use of LiTT in children.
Our results indicate that seizure reduction after LiTT compares favorably with conventional open surgical techniques. With an average follow-up of 20 months, 55\% of our patients have experienced greater than $90 \%$ reduction in seizures following the procedure; $50 \%$ are seizure free 2 years postoperatively. While the number of patients with at least 2 years of follow-up is limited in our series $(n=$ 8 ), those who were seizure free at 2 years have remained seizure free since surgery, and many have been weaned off medications, suggesting that favorable long-term outcome is possible with this technique. In a recent series of insular cortical resections in children published by Weil et al., $77 \%$ of patients had greater than $90 \%$ seizure reduction an average of 44 months after surgery ${ }^{16}$ Likewise, Malak et al. described 7 patients ( 6 adults and 1 adolescent) who underwent insular resection (5 right sided and 2 left sided) for intractable epilepsy. ${ }^{12}$ Only one underwent "pure" insulectomy, while the others required varying degrees of associated opercular and/or temporal lobe resection. At follow-up ranging from 14 to 122 months, all but 1 patient achieved seizure freedom. While seizure freedom was more favorable in these patients, $3(43 \%)$ sustained infarction within the corona radiata attributed to the surgery, and $4(57 \%)$ of the 7 patients experienced transient postoperative hemiparesis, which resolved over months.

Gras-Combe et al. reported on 6 adult patients (of whom 4 had normal findings on MRI) undergoing right transopercular insular resection after SEEG evaluation, of whom 5 at a mean follow-up of 36 months were rendered seizure free. ${ }^{8}$ All 6 of these patients were described as experiencing transient facial paresis or dysarthria. In our patients we did not note a difference in seizure-free outcome following pure insular ablation compared with ablation with opercular regions included. Only 7 (29\%) of our patients experienced postoperative functional deficits following LiTT with none persisting beyond 6 months, even when the opercular cortex was included in the target zone, suggesting that LiTT offers an alternative to open resection with less morbidity. Likewise, we did not note a difference in the occurrence of postoperative deficits related to the region (i.e., anterior vs posterior) of the insula that was treated with ablation. Despite temperature control settings, structures lateral to the putamen, often including the extreme capsule, claustrum, and external capsule, appeared to have been injured as depicted on delayed follow-up imaging (Fig. 1C and F); however, adverse effects were minimal. Although longitudinal (as opposed to orthogonal) placement of the laser catheter would seem to achieve wider insular coverage and ablation, the curved and "uneven" nature of the lateral insular surface entails a relatively medial trajectory to avoid crossing multiple pial surfaces and associated subarachnoid spaces. As a result, some of our "insular ablations" may, in fact, represent "insular disconnections," given the relationship of the laser fiber catheter just deep to the insular cortex. While seizure freedom may be less common after focused LiTT procedures compared with open resection, the decreased risk of adverse effects with still significant likelihood of favorable outcome makes LiTT an attractive consideration for initial surgical therapy.

LiTT compares favorably with radiofrequency ablation, another minimally invasive approach to insular surgery. 
Catenoix et al. described a series of 5 adult patients undergoing radiofrequency ablation of cortical malformations within the insula, which is "poorly accessible to surgical resection." ${ }^{\prime 3}$ Although 4 patients were described as responding to treatment and 1 as seizure free, 2 stopped responding to treatment at 2 and 6 months. Thus, LiTT appears to offer more sustained seizure reduction in a larger proportion of patients, although the number of patients treated remains too small to draw absolute conclusions.

This series is one of relatively few describing children undergoing LiTT for the treatment of epilepsy. ${ }^{4,11}$ Lewis et al. described their experience with 17 patients after LiTT for lesional intractable epilepsy. ${ }^{11}$ Forty-seven percent had a greater than $90 \%$ reduction in seizures at a mean of 16 months after therapy, similar to the outcomes presented in this series. The only other series of LiTT use in children, all with lesional epilepsy, consisted of 5 patients who underwent follow-up for 2-13 months after surgery. ${ }^{4}$ Our results are unique, not only because the insula was targeted for ablative treatment, but because $70 \%$ of our patients had normal findings on MRI. This suggests that LiTT can be effectively used in cases in which the findings are normal on MRI if the seizure onset zone is well delineated and of suitable size for ablation. Fifty-five percent of our patients with normal MRI findings were in Engel Class I or II postoperatively (mean follow-up 17.2 months, range 8-39 months).

In addition to comparing favorably with respect to seizure-free outcome and complications, LiTT requires shorter hospitalizations and thus would be expected to decrease the cost of care. Eighty-eight percent of our patients were discharged within 48 hours, and $63 \%$ were discharged within 1 day, similar to other series. ${ }^{11}$ Postoperative pain was minimal, as evidenced by only a few patients requiring narcotics. These factors enhance LiTT's appeal to patients and their parents, compared with more invasive techniques. In addition, encouraging outcomes and likely reduced cost of care suggest that LiTT should be initially offered to appropriate candidates, with more invasive resective procedures considered if LiTT fails.

Despite our favorable outcomes using LiTT for insular epilepsy, $20 \%$ of our patients experienced no appreciable improvement after surgery. This is likely attributable to the difficulty localizing the epileptogenic network in these cases, more so than a failure of LiTT. Of the patients in whom treatment failed, most either underwent numerous nonlocalized presurgical functional imaging and neurophysiology studies or had multiple lesions (i.e., tuberous sclerosis) on MRI, suggesting widespread epileptogenic zones. While our SEEG investigations suggested that the insula was the primary node of onset, the procedures that failed suggest that the network and our monitoring of that network were insufficient to completely characterize and treat the onset zone. We continue revising our methods for monitoring the insula with SEEG such that we adequately include the insula but also the perisylvian cortex for complete characterization of the epileptogenic zone.

This initial series of LiTT use in intractable insular epilepsy provides encouraging data supporting the efficacy of this approach in achieving seizure freedom with minimal adverse effects. Our small cohort reflects the relative rar- ity of truly insular epilepsy. While our follow-up period is short, the seizure-free outcome for those with at least 1 year of follow-up appears significant and has been maintained for those with at least 2 years of follow-up. The fact that several patients have been weaned off of all medications strongly suggests that this approach is effective for some patients. Longer follow-up and increased numbers of patients undergoing LiTT for intractable epilepsy will help provide a definition of appropriate candidates for this therapy. At present, LiTT may be considered an option for patients with well-localized seizure onset when the seizure onset zone is amenable in size and location to ablative treatment.

\section{Conclusions}

While rare, the insula is increasingly recognized as a cause of intractable localization-related epilepsy. Excision within the insula carries considerable risks given the extensive blood supply and proximity to critical white matter tracts. This surgical technique results in seizure-free outcomes that are comparable to open resection and requires only brief hospital stays with limited postoperative pain. These factors make LiTT an attractive option for patients and families considering surgical treatment for insular epilepsy and should be considered in appropriate candidates.

\section{References}

1. Afif A, Chabardes S, Minotti L, Kahane P, Hoffmann D: Safety and usefulness of insular depth electrodes implanted via an oblique approach in patients with epilepsy. Neurosurgery 62 (5 Suppl 2): ONS471-ONS480, 2008

2. Bouthillier A, Surbeck W, Weil AG, Tayah T, Nguyen DK: The hybrid operculo-insular electrode: a new electrode for intracranial investigation of perisylvian/insular refractory epilepsy. Neurosurgery 70:1574-1580, 2012

3. Catenoix H, Mauguière $\mathrm{F}$, Montavont $\mathrm{A}$, Ryvlin $\mathrm{P}$, Guénot $\mathrm{M}$, Isnard J: Seizures outcome after stereoelectroencephalography-guided thermocoagulations in malformations of cortical development poorly accessible to surgical resection. Neurosurgery 77:9-15, 2015

4. Curry DJ, Gowda A, McNichols RJ, Wilfong AA: MR-guided stereotactic laser ablation of epileptogenic foci in children. Epilepsy Behav 24:408-414, 2012

5. Dylgjeri S, Taussig D, Chipaux M, Lebas A, Fohlen M, Bulteau $\mathrm{C}$, et al: Insular and insulo-opercular epilepsy in childhood: an SEEG study. Seizure 23:300-308, 2014

6. Engel J Jr, Van Ness PC, Rasmussen TB, Ojemann LM: Outcome with respect to epileptic seizures, in Engel J Jr (ed): Surgical Treatment of the Epilepsies, ed 2. New York: Raven Press, 1993, pp 609-621

7. González-Martínez J, Bulacio J, Thompson S, Gale J, Smithason S, Najm I, et al: Technique, results, and complications related to robot-assisted stereoelectroencephalography. Neurosurgery 78:169-180, 2016

8. Gras-Combe G, Minotti L, Hoffmann D, Krainik A, Kahane $\mathrm{P}$, Chabardes S: Surgery for nontumoral insular epilepsy explored by stereoelectroencephalography. Neurosurgery 79:578-588, 2016

9. Isnard J, Guénot M, Sindou M, Mauguière F: Clinical manifestations of insular lobe seizures: a stereo-electroencephalographic study. Epilepsia 45:1079-1090, 2004

10. Kang JY, Wu C, Tracy J, Lorenzo M, Evans J, Nei M, et al: Laser interstitial thermal therapy for medically intractable mesial temporal lobe epilepsy. Epilepsia 57:325-334, 2016 
11. Lewis EC, Weil AG, Duchowny M, Bhatia S, Ragheb J, Miller I: MR-guided laser interstitial thermal therapy for pediatric drug-resistant lesional epilepsy. Epilepsia 56:1590-1598, 2015

12. Malak R, Bouthillier A, Carmant L, Cossette P, Giard N, Saint-Hilaire JM, et al: Microsurgery of epileptic foci in the insular region. J Neurosurg 110:1153-1163, 2009

13. Nguyen DK, Nguyen DB, Malak R, Leroux JM, Carmant L, Saint-Hilaire JM, et al: Revisiting the role of the insula in refractory partial epilepsy. Epilepsia 50:510-520, 2009

14. Silfvenius H, Gloor P, Rasmussen T: Evaluation of insular ablation in surgical treatment of temporal lobe epilepsy. Epilepsia 5:307-320, 1964

15. Türe U, Yaşargil MG, Al-Mefty O, Yaşargil DC: Arteries of the insula. J Neurosurg 92:676-687, 2000

16. Weil AG, Le NMD, Jayakar P, Resnick T, Miller I, Fallah A, et al: Medically resistant pediatric insular-opercular/perisylvian epilepsy. Part 2: outcome following resective surgery. J Neurosurg Pediatr 18:523-535, 2016

17. Willie JT, Laxpati NG, Drane DL, Gowda A, Appin C, Hao $\mathrm{C}$, et al: Real-time magnetic resonance-guided stereotactic laser amygdalohippocampotomy for mesial temporal lobe epilepsy. Neurosurgery 74:569-585, 2014

\section{Disclosures}

The authors report the following. Dr. Donahue: consultant for
Medtronic and Zimmer. Dr. Lee: consultant for Medtronic. Dr. Clarke: speakers' bureau for Lundbeck Pharmaceuticals and grant money from the Anderson Foundation.

\section{Author Contributions}

Conception and design: Perry, Clarke. Acquisition of data: all authors. Analysis and interpretation of data: Perry. Clarke Drafting the article: Perry, Donahue, Lee, Clarke Critically revising the article: all authors. Reviewed submitted version of manuscript: all authors. Approved the final version of the manuscript on behalf of all authors: Perry. Administrative/technical/material support: Perry. Study supervision: Perry.

\section{Supplemental Information}

Previous Presentations

A portion of this work was previously presented in abstract form at the American Society for Stereotactic and Functional Neurosurgery meeting, Chicago, IL, June 18-21, 2016.

\section{Correspondence}

M. Scott Perry, Comprehensive Epilepsy Center, Cook Children's Medical Center, 1500 Cooper St., 4th Fl., Fort Worth, TX 76104. email: scott.perry@cookchildrens.org. 